\title{
Osimertinib making a breakthrough in lung cancer targeted therapy
}

This article was published in the following Dove Press journal:

OncoTargets and Therapy

6 September 2016

Number of times this article has been viewed

\section{Haijun Zhang}

Department of Oncology, Zhongda Hospital, Medical School, Southeast University, Nanjing, People's Republic of China
Correspondence: Haijun Zhang Department of Oncology, Zhongda Hospital, Medical School, Southeast University, 87 Dingjiaqiao, Nanjing 210009, People's Republic of China Tel/fax +86 2583275416

Email zhanghaijunseu@।63.com

\begin{abstract}
Epidermal growth factor receptor (EGFR) tyrosine kinase inhibitors (TKIs) are the evidence-based first-line treatment for advanced non-small-cell lung cancer that harbors sensitizing $E G F R$ mutations $\left(E G F R \mathrm{~m}^{+}\right)$such as exon 19 deletions and L858R substitutions in exon 21. However, acquired resistance to EGFR TKIs is mostly driven by a second-site EGFR T790M mutation, which negates their inhibitory activity. Osimertinib (AZD9291, Tagrisso ${ }^{\mathrm{TM}}$ ), an oral, third-generation EGFR TKI, has been designed to target the EGFR T790M mutation, while sparing wild-type EGFR. In this up-to-date review, focus is not only on the structure, mechanisms, and pharmacokinetics of osimertinib but also on summarizing clinical trials and making recommendations of osimertinib for patients with non-small-cell lung cancer.
\end{abstract}

Keywords: osimertinib, epidermal growth factor receptor, tyrosine kinase inhibitors, non-small cell lung cancer, T790M mutation

\section{Introduction}

Lung cancer is the leading cause of cancer-related mortality worldwide, with $\sim 80 \%-85 \%$ of patients suffering from non-small-cell lung cancer (NSCLC) and a poor prognosis for those with advanced stage disease treated with traditional chemotherapy agents. ${ }^{1}$ The discovery of genetic drivers of NSCLC has demonstrated the power of precision medicine in genetically defined tumors, and the most important drivers are mutations in EGFR gene, which occur in $10 \%-35 \%$ of patients with NSCLC. "2,3 "Sensitizing mutations" in EGFR (EGFRm ${ }^{+}$) refer to exon 19 deletions and exon 21 L858R point mutations. Epidermal growth factor receptor (EGFR) tyrosine kinase inhibitors (TKIs)

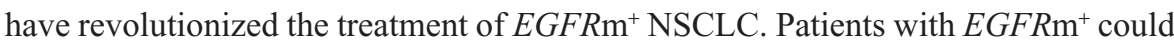
achieve good responses to therapy with the first-generation EGFR TKIs, such as erlotinib and gefitinib. ${ }^{4-6}$ Unfortunately, despite initial benefit, most patients develop drug resistance within 1 year, which is driven in $~ 60 \%$ of cases by a second-site $E G F R$ point mutation, the T790M mutation occurring within exon $20 .^{7,8}$ However, therapeutic strategies for patients with NSCLC with the acquired resistance mediated by T790M are still limited. Although second-generation EGFR TKIs, such as afatinib, dacomitinib, and neratinib, demonstrated promising activity against T790M in preclinical models, they have failed to overcome T790M-mediated resistance in patients due to dose-limiting toxicity resulting from nonselective inhibition of wild-type EGFR. ${ }^{9-11}$ Therefore, there remains a significant need for drugs that could more effectively target T790M while sparing wild-type EGFR. ${ }^{12}$ Recently, third-generation EGFR inhibitors, including osimertinib, WZ4002, and CO-1686, have been developed and shown to be effective against cell lines and murine models harboring T790M mutations while sparing wild-type EGFR. ${ }^{13}$ These mutant-selective inhibitors could represent a promising 


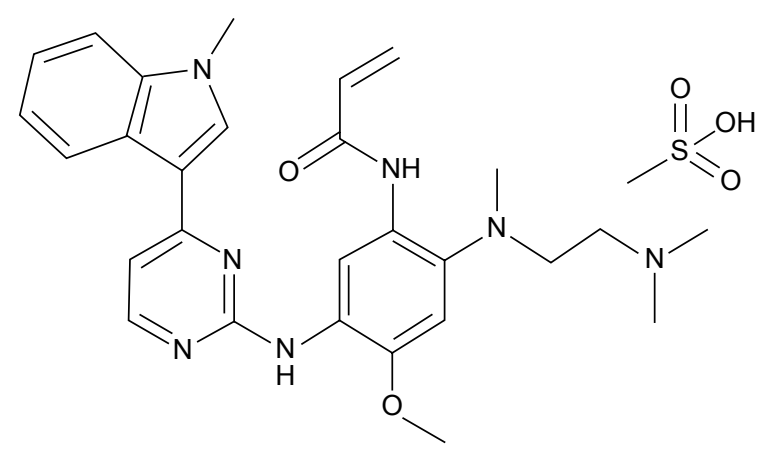

Figure I Chemical structure of osimertinib.

approach to overcome T790M-mediated resistance in patients with NCSLC.

Osimertinib, also known as AZD9291, is a novel EGFR TKI developed by AstraZeneca, the structure and pharmacology of which are distinct from other thirdgeneration EGFR TKIs. ${ }^{14}$ It shows 200 -fold selectivity for T790M/L858R protein over wild-type EGFR and has been classed as a "breakthrough" compound for fast-track development, having demonstrated perfect objective response rate (ORR) in patients with T790M-positive NSCLC who had progressed on a first-generation EGFR TKI. ${ }^{12}$ Osimertinib received its first global approval by the US Food and Drug Administration in November 2015 for patients with metastatic EGFR T790M-positive NSCLC who had progressed on prior systemic therapy, including an EGFR TKI.

In this review, the structure, mechanisms, and pharmacokinetics (PKs) of osimertinib are addressed. Clinical trials are also summarized and recommendations are made for osimertinib for patients with NSCLC.

\section{Structure and mechanism}

Osimertinib is a mono-anilino-pyrimidine small molecule. The molecular formula for osimertinib mesylate is $\mathrm{C}_{28} \mathrm{H}_{33} \mathrm{~N}_{7} \mathrm{O}_{2}$. $\mathrm{CH}_{4} \mathrm{O}_{3} \mathrm{~S}$ possessing a molecular weight of $596 \mathrm{~g} / \mathrm{mol}$, and its chemical name is $\mathrm{N}$-(2-\{2-dimethylaminoethyl-methylamino $\}$ 4-methoxy-5-\{[4-(1-methylindol-3-yl)pyrimidin-2yl]amino $\}$ phenyl)prop-2-enamide mesylate salt, which is shown in Figure 1. Osimertinib binds irreversibly, via the C797 amino acid covalent bond, to certain mutant forms of EGFR (L858R, exon 19 deletion, and double mutants containing T790M) at approximately ninefold lower concentrations than to wild-type EGFR. ${ }^{11}$ Then, several downstream pathways, such as RAS/ RAF/MAPK and PI3K/AKT, that regulate different cellular processes, including DNA synthesis and proliferation, could be inhibited, which is schematically illustrated in Figure 2. Thus, osimertinib acts as a novel and selective third-generation EGFR TKI to target the EGFR T790M mutation while sparing wild-type EGFR.

\section{Pharmacodynamics}

Osimertinib potently inhibited EGFR phosphorylation in both EGFR cells harboring EGFRm ${ }^{+}$and T790M mutant cell lines. But it was less potent at inhibiting phosphorylation of EGFR in wild-type cell lines. $\mathrm{IC}_{50}(50 \%$ inhibiting concentration) values for exon 19 deletion and L858R/T790M are $12.92 \mathrm{nM}$ and $11.44 \mathrm{nM}$, respectively, while $\mathrm{IC}_{50}$ for wild-type EGFR is $\sim 493.8 \mathrm{nM}^{11}$ This implies that osimertinib could more potently inhibit phospho-EGFR of L858R, exon 19 deletion, and double mutants containing T790M compared with wildtype EGFR. ${ }^{11}$ AZ7550 and AZ5104 are the two pharmacologically active metabolites of osimertinib. The former has a very

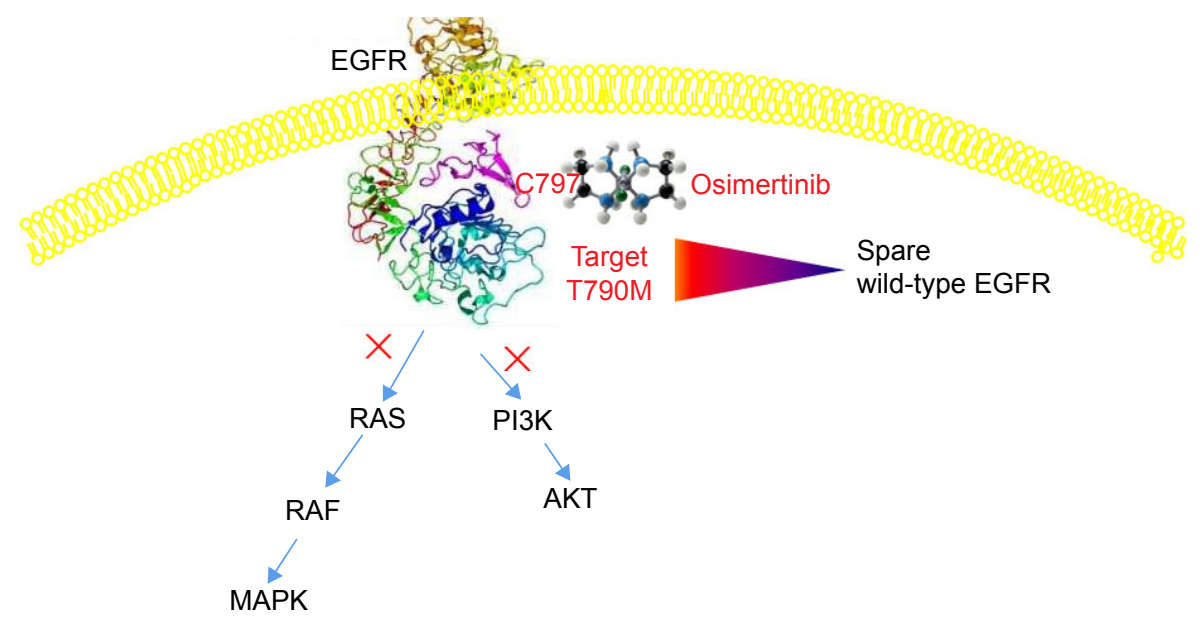

Figure 2 Schematic illustration of the possible mechanism of osimertinib.

Notes: Via the C797 amino acid covalent bond, osimertinib could target the EGFR T790M mutation while sparing wild-type EGFR, thus inhibiting several downstream pathways, such as RAS/RAF/MAPK and PI3K/AKT, that regulate different cellular processes, including DNA synthesis and proliferation.

Abbreviation: EGFR, epidermal growth factor receptor. 
similar profile to osimertinib, but the latter exhibits a reduced selectivity margin against wild-type EGFR compared with the parent drug. ${ }^{11}$ Consistently, patients with detectable plasma levels of EGFR T790M DNA had a greater than twofold higher clinical response rate than those without detectable plasma ( $85 \%$ vs $33 \%$ ) when osimertinib was the second-line treatment for patients with EGFRm ${ }^{+}$NSCLC. ${ }^{15}$

\section{Pharmacokinetics}

Osimertinib was slowly absorbed and displayed doseproportional increases in exposure from $20 \mathrm{mg}$ to $240 \mathrm{mg}$. ${ }^{16,17}$ Distribution was extensive and clearance was low to moderate with a mean half-life of 48.3 hours. Steady state could be reached after 15 days, consistent with single-dose PK. At steady state, the $C_{\max }$ to $C_{\min }$ ratio of osimertinib was 1.6-fold. ${ }^{17}$ Two active metabolites, AZ7550 and AZ5104, circulated at $\sim 10 \%$ of osimertinib exposure. Age, sex, bodyweight, ethnicity, mild or moderate renal impairment, or mild hepatic impairment does not affect the PK profile of osimertinib. Food caused a clinically insignificant increase in exposure.

\section{Preclinical research}

Preclinical studies have been dedicated to the antitumor activity of osimertinib in vitro and in vivo. ${ }^{3,11}$ In vitro, osimertinib potently inhibits signaling pathways and cellular growth in both $E G F R \mathrm{~m}^{+}$and $E G F R \mathrm{~m}^{+} / \mathrm{T} 790 \mathrm{M}$ mutant cell line, with lower activity against wild-type $E G F R$ lines. It also inhibits the activity of ERBB2, ERBB3, ERBBR4, ACK1, and BLK at clinically relevant concentrations. Encouraged by the in vitro remarkable inhibitory activity, the investigation of the potential antitumor effect of osimertinib in vivo was further performed. In addition, the results demonstrated that osimertinib also caused profound and sustained tumor regression in tumor xenograft and transgenic mouse models harboring activating EGFR mutations and EGFR T790M.

\section{Clinical trials}

The efficacy of osimertinib for the treatment of patients with locally advanced or metastatic EGFRm ${ }^{+}$NSCLC has been investigated in several clinical trials.

\section{Phase I}

The Phase I AURA trial (NCT01802632) was initiated to assess the safety and efficacy of osimertinib in patients with locally advanced or metastatic EGFRm ${ }^{+}$NSCLC who had documented disease progression while receiving a first- or second-generation EGFR TKI. ${ }^{16}$ A total of 253 patients were enrolled. The study included two cohorts: the dose-escalation cohort with 31 patients and the dose-expansion cohort with 222 patients who received oral osimertinib at five dosages of $20 \mathrm{mg} / \mathrm{d}, 40 \mathrm{mg} / \mathrm{d}, 80 \mathrm{mg} / \mathrm{d}, 160 \mathrm{mg} / \mathrm{d}$, or $240 \mathrm{mg} / \mathrm{d}$. Among 31 patients enrolled in the dose-escalation cohorts, no dose-limiting toxic effects were observed. An additional 222 patients were treated in five expansion cohorts. The ORR was 51\% (95\% confidence interval [CI], 45-58). Of the 127 patients with confirmed EGFR T790M, osimertinib was associated with an ORR of $61 \%$ (95\% CI, 52-70), while those with no detectable EGFR T790M mutation (n=61) had an ORR of $21 \%$ (95\% CI, 12-34). The median progressionfree survival (PFS) was 9.6 months (95\% CI, 8.3 to not reached) in EGFR T790M-positive patients and 2.8 months (95\% CI, 2.1-4.3) in EGFR T790M-negative patients. Tumor response rates were similar across all osimertinib dose levels, with increasing toxicities at the $160 \mathrm{mg}$ and $240 \mathrm{mg}$ daily doses; thus, a dose of $80 \mathrm{mg}$ daily was adopted for future studies. ${ }^{18}$ Based on these data, in April 2014, osimertinib was granted breakthrough therapy designation by the US Food and Drug Administration for the treatment of patients with NSCLC and EGFR T790M mutation whose disease has progressed during treatment with a TKI.

\section{Phase II}

Based on the abovementioned Phase I findings, osimertinib was further evaluated in EGFR T790M mutation-positive NSCLC patients who progressed after EGFR TKIs in a Phase II extension cohort of AURA (NCT01802632) and an additional Phase II trial (AURA2; NCT02094261). A total of 411 patients were assigned to treatment in the AURA extension trial $(n=201)$ and the AURA2 trial $(n=210)$. Results from a November 1, 2015, data cutoff for pooled analysis of two Phase II studies showed that ORR was 66\% (95\% CI, 61-71), median duration of remission was 12.5 months (95\% CI: 11.1 to not calculable), median PFS was 11.0 months (95\% CI: 9.6-12.4), and the proportion of patients who were progression free at 12 months was $47.5 \%$ (95\% CI: 42.4-52.5). ${ }^{19}$ Thus, osimertinib received its first global approval on November 13, 2015, for patients with metastatic EGFR T790M mutation-positive NSCLC who have progressed on or after EGFR TKI therapy in the US.

\section{Ongoing Phase III studies}

Several Phase III trials are ongoing in evaluating osimertinib as second-line treatment for EGFR T790M-mutated NSCLC as well as first-line treatment for NSCLC with any EGFRm ${ }^{+}{ }^{15}$ From August 2014 to December 2017, the AURA3 trial (NCT02151981), an open-label, randomized 
study with a primary end point of PFS, investigates osimertinib versus platinum-based doublet chemotherapy as second-line therapy for locally advanced or metastatic NSCLC with EGFR T790M mutation, in which patients in the chemotherapy arm who develop progressive disease will be eligible to cross over to osimertinib treatment. The FLAURA trial (NCT02296125) from December 2014 to October 2018 is a randomized, double-blind, multinational, Phase III trial with a primary end point of PFS, which studies osimertinib versus gefitinib or erlotinib as first-line therapy in treatment-naïve patients with locally advanced or metastatic $E G F R \mathrm{~m}^{+} \mathrm{NSCLC}$. For adjuvant therapy, the ADAURA trial (NCT02511106) from October 2015 to September 2021 is a randomized, double-blind, multinational Phase III trial with the primary end point of disease-free survival to compare the efficacy and safety of osimertinib with that of placebo in patients with EGFR mutation-positive stage Ib-IIIa NSCLC following complete tumor resection. Besides NSCLC, there is one clinical trial to investigate the PKs, safety, and tolerability of osimertinib in patients with advanced solid tumors and mild or moderate hepatic impairment or normal hepatic function (NCT02161770).

\section{Safety and side effects}

Osimertinib has a good tolerability profile in patients with locally advanced or metastatic EGFR mutation-positive NSCLC. ${ }^{16}$ There were no dose-limiting toxicities observed in patients in the dose-escalation cohort of AURA at any dose level ranging from $20 \mathrm{mg}$ to $240 \mathrm{mg}$ daily during a 28-day evaluation period, and therefore the maximum tolerated dosage was not defined. Among the combined cohort of 253 patients in the trial, the most common adverse events included diarrhea (47\%), rash (40\%), nausea (22\%), and decreased appetite (21\%). Any adverse event of grade 3 or higher occurred in $32 \%$ of patients, of which $13 \%$ were drug related.

\section{Conclusion and future directions}

The EGFR T790M mutation accounts for the majority of acquired resistance to first- and second-generation EGFR TKIs and represents a significant obstacle in the treatment of EGFRm ${ }^{+}$advanced NSCLCs. ${ }^{20}$ The third-generation EGFRTKIs were designed and developed to inhibit the EGFR T790M mutation while sparing wild-type EGFR, such as osimertinib, rociletinib (CO-1686), and olmutinib (HM61713). In the early-phase clinical trials, the ORRs of osimertinib, rociletinib, and olmutinib are $64 \%, 58 \%$, and $29.2 \%$, respectively. ${ }^{21}$ Osimertinib is a covalent third-generation
EGFR TKI with significant activity against the EGFR T790M resistance mutation and common EGFR-activating mutations, and low activity against wild-type EGFR. Phase I and II studies of osimertinib have shown ORR ranging from $57 \%$ to $66 \%$ with well-tolerated profile. So far, osimertinib is the first global EGFR TKI approved for the treatment of NSCLC with EGFR T790M mutation. The main focus of academic research moving forward for osimertinib will hinge on preventing acquired resistance to monotherapy and attempting to combine osimertinib with other anticancer therapies.

\section{Acknowledgment}

This work was supported by the National Natural Science Foundation of China (81371678).

\section{Disclosure}

The author reports no conflicts of interest in this work.

\section{References}

1. Zhang H. Apatinib for molecular targeted therapy in tumor. Drug Des Devel Ther. 2015;9:6075-6081.

2. Russo A, Franchina T, Ricciardi GR, et al. A decade of EGFR inhibition in EGFR-mutated non small cell lung cancer (NSCLC): old successes and future perspectives. Oncotarget. 2015;6(29):26814-26825.

3. Hirano T, Yasuda H, Tani T, et al. In vitro modeling to determine mutation specificity of EGFR tyrosine kinase inhibitors against clinically relevant EGFR mutants in non-small-cell lung cancer. Oncotarget. 2015;6(36):38789-38803.

4. Tiseo M, Bartolotti M, Gelsomino F, Bordi P. Emerging role of gefitinib in the treatment of non-small-cell lung cancer (NSCLC). Drug Des Devel Ther. 2010;4:81-98.

5. Ren Y, Yao Y, Ma Q, Zhong D. EGFR gene-mutation status correlated with therapeutic decision making in lung adenocarcinoma. Onco Targets Ther. 2015;8:3017-3020.

6. Zhang Q, Wang Z, Guo J, et al. Comparison of single-agent chemotherapy and targeted therapy to first-line treatment in patients aged 80 years and older with advanced non-small-cell lung cancer. Onco Targets Ther. 2015;8:893-898.

7. Ercan D, Choi HG, Yun CH, et al. EGFR mutations and resistance to irreversible pyrimidine-based EGFR inhibitors. Clin Cancer Res. 2015; 21(17):3913-3923.

8. Villadolid J, Ersek JL, Fong MK, Sirianno L, Story ES. Management of hyperglycemia from epidermal growth factor receptor (EGFR) tyrosine kinase inhibitors (TKIs) targeting T790M-mediated resistance. Transl Lung Cancer Res. 2015;4(5):576-583.

9. Ramalingam SS, Blackhall F, Krzakowski M, et al. Randomized phase II study of dacomitinib (PF-00299804), an irreversible pan-human epidermal growth factor receptor inhibitor, versus erlotinib in patients with advanced non-small-cell lung cancer. J Clin Oncol. 2012;30(27): 3337-3344.

10. Sequist LV, Yang JC, Yamamoto N, et al. Phase III study of afatinib or cisplatin plus pemetrexed in patients with metastatic lung adenocarcinoma with EGFR mutations. J Clin Oncol. 2013;31(27):3327-3334.

11. Cross DA, Ashton SE, Ghiorghiu S, et al. AZD9291, an irreversible EGFR TKI, overcomes T790M-mediated resistance to EGFR inhibitors in lung cancer. Cancer Discov. 2014;4(9):1046-1061.

12. Yosaatmadja Y, Silva S, Dickson JM, et al. Binding mode of the breakthrough inhibitor AZD9291 to epidermal growth factor receptor revealed. J Struct Biol. 2015;192(3):539-544. 
13. Pirker R. Third-generation epidermal growth factor receptor tyrosine kinase inhibitors in advanced nonsmall cell lung cancer. Curr Opin Oncol. 2016;28(2):115-121.

14. Jiang T, Zhou C. Clinical activity of the mutant-selective EGFR inhibitor AZD9291 in patients with EGFR inhibitor-resistant non-small cell lung cancer. Transl Lung Cancer Res. 2014;3(6):370-372.

15. Greig SL. Osimertinib: first global approval. Drugs. 2016;76(2): 263-273.

16. Jänne PA, Yang JC, Kim DW, et al. AZD9291 in EGFR inhibitor-resistant non-small-cell lung cancer. N Engl J Med. 2015;372(18):1689-1699.

17. Planchard D, Brown KH, Kim DW, et al. Osimertinib Western and Asian clinical pharmacokinetics in patients and healthy volunteers: implications for formulation, dose, and dosing frequency in pivotal clinical studies. Cancer Chemother Pharmacol. 2016;77(4):767-776.
18. Gao X, Le X, Costa DB. The safety and efficacy of osimertinib for the treatment of EGFR T790M mutation positive non-small-cell lung cancer. Expert Rev Anticancer Ther. 2016;16(4):383-390.

19. Yang J, Ramalingam SS, Jänne PA, Cantarini M, Mitsudomi T. LBA2_PR: osimertinib (AZD9291) in pre-treated pts with T790Mpositive advanced NSCLC: updated phase 1 (P1) and pooled phase 2 (P2) results. J Thorac Oncol. 2016;11(4 suppl):S152-S153.

20. Li H, Hu H, Wang R, et al. Primary concomitant EGFR T790M mutation predicted worse prognosis in non-small cell lung cancer patients. Onco Targets Ther. 2014;7:513-524.

21. Liao BC, Lin CC, Yang JC. Second and third-generation epidermal growth factor receptor tyrosine kinase inhibitors in advanced nonsmall cell lung cancer. Curr Opin Oncol. 2015;27(2):94-101.

\section{Publish your work in this journal}

OncoTargets and Therapy is an international, peer-reviewed, open access journal focusing on the pathological basis of all cancers, potential targets for therapy and treatment protocols employed to improve the management of cancer patients. The journal also focuses on the impact of management programs and new therapeutic agents and protocols on

\section{Dovepress}

patient perspectives such as quality of life, adherence and satisfaction. The manuscript management system is completely online and includes a very quick and fair peer-review system, which is all easy to use. Visit http://www.dovepress.com/testimonials.php to read real quotes from published authors.

Submit your manuscript here: http://www.dovepress.com/oncotargets-and-therapy-journal 